EESTI NSV TEADUSTE AKADEEMIA TOIMETISED. XIII KOIDE FOOSIKA-MATEMAATIKA- JA TEHNIKATEADUSTE SEERIA. 1964, NR. 3

ИЗВЕСТИЯ АКАДЕМИИ НАУК ЭСТОНСКОИ ССР. ТОМ ХІІІ

СЕРИЯ ФИЗИКО-МАТЕМАТИЧЕСКИХ И ТЕХНИЧЕСКИХ НАУК. 1964, № 3

\title{
К ТЕОРИИ ШИРИНЫ ЧИСТОЭЛЕКТРОННОЙ ЛИНИИ И ЛИНИИ МЕССБАУЭРА СВОБОДНОИ МОЛЕКУЛЫ
}

\author{
K. РЕБАНЕ, \\ член-корреспондент Академии наук Эстонской ССР
}

о. сильд

В работе иселедовано влияние поступательных, вращательных и колебательных степеней свободы на ширину чистоэлектронной спектральной линии и линии Мессбауэра свободной молекулы. Показано, что по мере возрастания массы молекулы ширина гпектральной линии уменьшается, причем начинает преобладать (допплеровское) уширение за счет поступательных степеней свободы, а в пределе весьма больших молекул (кристаллов) спектральная линия становится настоящей чистоэлектронной линией или настоящей линией Мессбауэра. Выяснены условия экспериментального обнаружения узкой чистоэлектронной линии в спектрах поглощения или излучения больших молекул: при комнатной температуре ширина линии будет менье $1 \mathrm{~cm}^{-1}$ у молекул с массой больше $10^{4} m_{H}$.

\section{1. Введение}

Цель настоящей работы - дать теоретическую оценку ширины чистоэлектронной линии и-линии Мессбауэра свободной молекулы. Наш подход заключается в прослеживании влияния поступательных, вращательных и колебательных степеней свободы на ширину линий по мере возрастания массы молекулы. В пределе весьма больших масс «молекулы» мы приходим, очевидно, к кристаллу, в случае которого линия $\gamma$-кванта становится «настоящей» чрезвычайно узкой резонансной линией Мессбауэра, а чистоэлектронная линия - оптическим аналогом этой линии $\left[{ }^{1-4}\right]$. Такой подход представляется нам полезным для более глубокого понимания эффекта Мессбауэра, эффекта Шпольского $[5,6,2]$ и других оптических аналогов эффекта Мессбауэра. Кроме того, мы приходим к выводу о теоретической возможности экспериментального обнаружения сравнительно узких чистоэлектронных линий в спектрах поглощения или люминесценции больших молекул. Осуцествление соответствуюших экспериментов дало бы ценную добавочную информацию об электронных состояниях сложных молекул, о степени выполнимости адиабатического приближения и электронно-колебательном взаимодействии в них.

Мы будем исследовать в отдельности вклады от поступательного, вращательного и колебательного движений ядер молекул в распределение вероятностей электронного и внутриядерного переходов. Разделение поступательного, вращательного и колебательного движений ядер проведем в адиабатическом приближении. В этом приближении распре- 
деление вероятностей поглощения или излучения фотона определяется в основном (точнее см., например [7]) квадратами матричных элементов

$$
M_{f i}=\int \Psi_{11 f}^{*}(X) M(X) \Psi_{1 i}(X) d X
$$

где $M(X)$ - матричный элемент электронного или внутриядерного перехода в молекуле; $\Psi_{\text {IIf }}(X)$ и $\Psi_{I i}(X)$ - собственные функции операторов Гамильтона $\widehat{H}_{\mathrm{II}}$ и $\widehat{H}_{\mathrm{I}}$, описывающих в адиабатическом приближении ядерное движение в конечном и исходном состояниях; $X$ - совокупность ядерных координат.

Матричный элемент электронного перехода имеет вид

$$
M(X)=\int d x \Phi_{\mathrm{II}}^{*}(x, X)\left\{\sum_{l} \frac{e_{l}}{m_{l}} \exp \left(-i \vec{K} \vec{R}_{l}\right)\left(\vec{e}_{P_{l}}\right)\right\} \Phi_{\mathrm{I}}(x, X),
$$

где $\Phi_{I I}(x, X)$ и $\Phi_{\mathrm{I}}(x, X)$ - электронные волновые функции адиабатического приближения в конечном и исходном электронных состояниях; $e_{l}$ и $m_{l}$ - заряд и масса частицы номера $l ; \vec{R}_{l}$ и $\vec{P}_{l}-$ радиус-вектор и оператор импульса частицы номера $l ; \vec{K}$ - волновой вектор фотона $(|\vec{K}|=\omega / c) ; \vec{e}$ - вектор поляризации фотона; $x$ - совокупность электронных координат. Ввиду ортогональности электронных волновых функций адиабатического приближения, при рассмотрении перехода с изменением электронного состояния сумму по $l$ следует брать только по электронам.

Матричный элемент внутриядерного перехода, фигурирующий в интегралах, определяющих распределение вероятностей переходов по подуровням движения ядер молекулы, имеет вид [ $\left.{ }^{8}\right]$

$$
M(X)=\exp \left(\overrightarrow{i K} \vec{R}_{L}\right)
$$

где $\vec{R}_{L}$ - радиус-вектор ядра номера $L$ : в котором происходит при поглощении или излучении $\gamma$-кванта ядерный переход.

Функция распределения $I(\Delta E)$ вероятностей $\left|M_{f i}\right|^{2}$ в шкале энергий перехода $\Delta E$ дается выражением

$$
I(\Delta E)=\sum_{i} n_{i} \sum_{f}\left|M_{f i}\right|^{2} \delta\left(E_{\mathrm{II} f}-E_{1 i}-\Delta E\right)
$$

где $n_{i}$ - вероятность нахождения молекулы в исходном состоянии $i$. Функцию $I(\Delta E)$ можно представить в виде интеграла

$$
I(\Delta E)=\frac{1}{2 \pi \hbar} \int d t \exp \left(-\frac{i \Delta E t}{\hbar}\right) I(t)
$$

где характеристическая функция $I(t)$ равна [9]

$$
I(t)=\frac{S p\left[M^{+} \exp \left(\frac{i \widehat{H}_{\mathrm{II}} t}{\hbar}\right) M \exp \left(-\frac{i \widehat{H}_{\mathrm{I}} t}{\hbar}\right) \exp \left(-\frac{\widehat{H}_{\mathrm{I}}}{k T}\right)\right]}{S p\left[\exp \left(-\frac{\widehat{H}_{\mathrm{I}}}{k T}\right)\right]}
$$


Қоэффициенты разложения $I(t)$ в степенной ряд представляют собой моментн функции распределения вероятностей $I(\Delta E)$ [9]. Прямой вывод формул моментов, с помощью которых можно провести рассмотрение, свободное от некоторых сделанных ниже упрощений, дан в работах $\left[10,{ }^{11}\right]$ Для наших целей, однако, нет здесь необхо димости в более точном расчете.

Для разделения поступательного, вращательного и колебательного движений ядер молекулы представим оператор Гамильтона $\widehat{H}$, описывающий в адиабатическом приближении ядерное движение в определенном электронном или внутриядерном состоянии, во вспомогательной системе координат $x y z$, прикрепленной к молекуле и вращающейся вместе $с$ ней. Начало системы координат. $O$ находится в центре тяжести молекулы. Направления осей $x, y, z$ огносительно движующейся поступательно вместе с центром тяжести молекулы системы координат $X^{\prime} Y^{\prime} Z^{\prime}$ определяются эйлеровыми углами из условий, чтобы момент количества движения молекулы с фиксированными в равновесных положениях ядрами был равен нулю относительно системы координат $x y z$ (см., например $\left.\left[{ }^{12}\right]\right)$. При таком выборе осей три декартовые координаты молекулы в системе координат наблюдателя $X Y Z$ описывают поступательное движение центра тяжести молекулы, три эйлеровых угла - вращение молекулы, а колебательное движе ние молекулы рассматривается при помощи $3 N-6$ (в случае линейной молекулы $3 N-5$ ) независимых ядерных координат в системе координат хуz, относительно которой молекула уже не совершает ни поступательного, ни вращательного движений (здесь $N$ число ядер в молекуле).

Оператор Гамильтона имеет в этих координатах следующий вид (см. формулу (48), главы XI, книги $\left[{ }^{[2]}\right]$ ):

$$
\begin{gathered}
\widehat{H}=\frac{1}{2 m}\left(P_{X}^{2}+P_{Y}^{2}+P_{Z}^{2}\right)+ \\
+\frac{1}{2} \mu^{\frac{1}{4}} \sum_{\gamma, \delta}\left(M_{\gamma}-m_{\eta}\right) \mu_{\gamma \delta} \mu^{-\frac{1}{2}}\left(M_{\hat{\zeta}}-m_{\delta}\right) \mu^{\frac{1}{4}}+\frac{1}{2} \mu^{\frac{1}{4}} \sum_{k} P_{k} \mu^{-\frac{1}{2}} P_{k} \mu^{\frac{1}{4}}+V(Q) .
\end{gathered}
$$

Обозначения: $m$ - масса молекулы; $X, Y, Z$ - декартовы координаты центра тяжести молекулы; $P_{X}, P_{Y}, P_{Z}$ - сопряженные им импульсы; $M_{\gamma}$ - компоненты момента количества движения молекулы в вращающейся системе координат; $m_{\text {. }}$ - компоненты момента количества движения, связанного с колебаниями молекулы; $\mu$ - определитель матрицы коэффициентов $\mu_{\gamma}$ (являющихся функциями колебательных коорди. нат $Q_{k}$ ) классического выражения кинетической энергии вращения молекулы $\left(\frac{1}{2}\right) \sum_{\gamma, \delta}\left(M_{\gamma}-m_{\gamma}\right) \mu_{\gamma \delta}\left(M_{\delta}-m_{\delta}\right) ; P_{k}-$ сопряженный $k$-той колебательной нормальной координате $Q_{k}$ импульс; $V$ - потенциальная энергия молекулы; $Q-$ совокупность нормальных координат $Q_{k}$.

Первая сумма в операторе Гамильтона (7) определяет энергию поступательного движения молекулы. Вторая сумиа определяет энергию врашательного движения молекулы вместе с взаимодействием моментов количества движения, соответствующих вращению и колебанию (эффект Қориолиса). Остальные члены относятся к кинетической и потенциальной энергий колебаннй молекулы в ненодвижной относительно ее системе координат.

В предположении малых колебаний коэффициенты $\mu_{\gamma \delta}$ можно считать независяцими от координат $Q_{k}$. Если пренебречь моментом количества движения, соответстнующим колебанию, а тем самым и взаимодействием между вращением и колебанием молекулы, то энергия вращательного движения выражается следующим простым образом:

$$
\frac{1}{2}\left(\frac{M_{x}^{2}}{I_{x}^{0}}+\frac{M_{y}^{2}}{I_{y}^{0}}+\frac{M_{z}^{2}}{I_{z}^{0}}\right)
$$

где $I_{x}^{0}, I_{y}^{0}, I_{z}^{0}$ - главные моменты ннерцин молекулы с фиксированными в равновесных положениях ядрамн. 


\section{2. Поступательное движение}

Для исследования вклада от поступательного движения молекулы в распределение вероятностей переходов фиксируем исходное и конечное электронно-колебательно-вращательные (или внутриядерно-колебательно-вращательные) состояния. Будем вычислять функцию распределения вероятностей $I(\Delta E)$ для электронно-колебательно-вращательного (внутриядерно-колебательно-вращательного) перехода между данными фиксированными состояниями. Распределение по степеням свободы поступательного движения молекул в исходном состоянии считаем максвелловским.

Проинтегрировав в (1) по колебательным и вращательным координатам, получим

$$
M_{f i}^{\text {пост }}=\int \varphi_{f}^{*}(X, Y, Z) M(X, Y, Z) \varphi_{i}(X, Y, Z) d X d Y d Z
$$

где $M(X, Y, Z)$ - матричный элемент фиксированного выше перехода; $\varphi_{f}$ и $\varphi_{i}-$ собственные функции оператора энергии поступательного движения. (9) представляет собой матричный элемент перехода $i \rightarrow f$ по поступательным степеням свободы.

Представив матричные элементы (2) и (3) в системе координат $X^{\prime} Y^{\prime} Z^{\prime}$, их зависимость от координат поступательного движения выделяется в виде множителя $\exp (\overrightarrow{i K R})(\vec{R}$ - радиус-вектор центра тяжести молекулы). Следовательно, матричный элемент $M(X, Y, Z)$ имеет в случае как электронного, так и ядерного перехода следующий вид:

$$
M(X, Y, Z) \sim \exp (i \vec{K} \vec{R})
$$

Матричный элемент $M_{f i}^{\text {пост }}$ равен $M_{f i}^{\text {пост }}=\delta\left(\vec{P}_{f}-\vec{P}_{i}-\hbar \vec{K}\right)$ и выражает сохранение импульса при поглощении или при излучении ( $九 \vec{K}$ импульс фотона). При поглощении фотона в молекуле ее импульс увеличится на импульс фотона $\hbar \vec{K}$, а при излучении фотона молекулой ее импульс увеличится на импульс отдачи - $\vec{K}$. Дальше мы прямо вычислим функцию распределения вероятностей $\left|M_{f i}^{\text {пост }}\right| 2$.

Для вычисления функции распределения вероятностей $\|\left. M_{f i}^{\text {пост }}\right|^{2}$ используем формулы (5) и (6), принимая в них $M=\exp (i \overrightarrow{K R} \vec{R}$ и $\widehat{H}_{\mathrm{II}}=\widehat{H}_{\mathrm{I}}=\left(P_{X}^{2}+P_{Y}^{2}+P_{Z}^{2}\right) / 2 m$ (мы считаем, что масса молекулы $m$ практически не изменяется в результате перехода).

В шкале энергин перехода поступательного движения $\Delta E^{\text {пост }}$ результат следующнй:

$$
I\left(\Delta E^{\text {nоcт }}\right)=\frac{1}{\sqrt{\pi \cdot 4 E_{R} k T}} \exp \left[-\frac{\left(\Delta E^{\text {nост }}-E_{R}\right)^{2}}{4 E_{R} k T}\right],
$$

где $E_{R}=(\hbar \omega)^{2} / 2 m c^{2}-$ энергия отдачи, сообщаемая фотоном молекуле. Используя сохранение энергии $-\hbar \omega+\Delta E^{\text {пост }}+E_{0}=0$ при поглощенин и $\hbar_{\omega}+\Delta E^{\text {пост}}-E_{0}=0$ при излучении $\left(E_{0}-\right.$ величина энергии перехо- 
да по всем другим степеням свободы), получим распределение вероятностей в шкале наблюдаемых частот в виде следующей формулы:

$$
I(\hbar \omega)=\frac{1}{\sqrt{\pi \cdot 4 E_{R} k T}} \exp \left[-\frac{\left(\hbar \omega-E_{0} \pm E_{R}\right)^{2}}{4 E_{R} k T}\right]
$$

где знак «+» относится к излучению, а: « к поглощению. Сохранение импульса, как уже отмечалось, содержится в самих матричных элементах $M_{f i}^{\text {noct }}$.

В хорошем приближении можно считать $E_{R} \approx(\hbar \bar{\omega})^{2} / 2 m c^{2}=$ const $(\bar{\omega}$ - средняя наблюдаемая частота), после чего (12) принимает вид обычной формулы, описывающей допплеровское уширение линии. Любопытно отметить, что мы получили ее без рассмотрения эффекта Допплера, исходя из картины стационарных квантовых состояний.

Распределение вероятностей по (12) имеет максимум при $\hbar \omega=$ $=E_{0}-\left( \pm E_{R}\right)$ и полуширину $\delta=4 \sqrt{E_{R} k T \ln 2}$. Линии поглощения и излучения смешены относительно друг друга на величину $2 E_{R}$ (сравни со стоксовыми смещениями электронно-колебательных спектров). На рисунке приведены зависимости полуширины $\delta$ и смещения $2 E_{R}$ от массы молекулы и энергии фотона при комнатной температуре (см. рисунок, кривые 1 и 2).

\section{3. Вращательное движение}

Для исследования вклада от степеней свободы вращательного движения молекулы в распределение вероятностей переходов, фиксируем опять исходное и конечное состояния по другим степеням свободы и будем вычислять функцию распределения вероятностей для перехода между фиксированными таким образом группами состояний.

Будем рассматривать линейные молекулы или молекулы типа сфернческого волчка. Для линейных молекул $I_{x}^{0}=I_{y}^{0}=I ; I_{z}^{0}=0$ и для мотекул типа сферического волчка $I_{x}^{0}=I_{y}^{0}=I_{z}^{0}=I$. Пренебрегая взаимодействием между колебаниями и вращениями, мы получаем согласно (8) энергию вращения в виде $\widehat{M}^{2} / 2 I$, где $\widehat{M}$ - момент количества движения.

После интегрирования в (1) по всем ядерным координатам, кроме трех вращательных, получим матричный элемент перехода $i \rightarrow f$ ло вращательным степеням свободы в виде

$$
M_{f i}^{\text {вращ }}=\int d X^{\text {вращ }} \psi_{f}^{*}\left(X^{\text {вращ })} M\left(X^{\text {вращ }}\right) \psi_{i}\left(X^{\text {вращ }}\right),\right.
$$

где $\psi_{i}\left(X^{\text {вращ }}\right)$ н $\psi_{i}\left(X^{\text {враш }}\right)$ - собственные функции оператора $\widehat{M}^{2} / 2 I$.

Для электронного перехода будем использовать матричный эле мент [ $\left.{ }^{2}\right]$ в дипольном приближении

$$
\begin{gathered}
M(X)=\int d x \Phi_{\mathrm{II}}^{*}(x, X)\left(\sum_{l} \frac{e_{l}}{m_{l}} \vec{e} \vec{P}_{l}\right) \Phi_{\mathrm{I}}(x, X)= \\
=\frac{i}{\hbar}\left[E_{\mathrm{II}}(X)-E_{\mathrm{I}}(X)\right] \int d x \Phi_{\mathrm{II}}^{*}(x, X)(\vec{e} \vec{D}) \Phi_{\mathrm{I}}(x, X),
\end{gathered}
$$

где $\vec{D}=\sum_{l} e_{l} \vec{R}_{l}-$ дипольный момент электронов молекулы; $E_{11}(X)$ 
$E_{1}(X)$ - собственные значения электронного уравнения адиабатического приближения. Ниже мы будем интересоваться распределениен квадратов матричных элементов

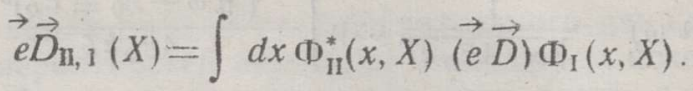

Во введенных выше приближениях проще описать вращение в движущейся поступательно вместе с центром тяжести молекулы системе сфе: рических координат $r, \vartheta, \varphi$. Тогда

$$
M\left(X^{\text {враш }}\right)=\left\langle\vec{D}_{11,1}\right\rangle\left(e_{X^{\prime}} \sin \vartheta \cos \varphi+e_{Y^{\prime}} \sin \vartheta \sin \varphi+e_{Z} \cdot \cos \vartheta\right),
$$

где $\left\langle\vec{D}_{\mathrm{ll}, \mathrm{I}}\right\rangle$ означает матричный элемент дипольного момента по колебательным состояниям, который мы здесь считаем независящим от вращательного состояния.

Для вычисления матричных элементов $M_{f i}^{\text {вращ }}$ в этом приближении мы приходим к интегралам, встречающимся при вычислении правил отбора для углового момента атома и можем непосредственно пользоваться обшензвестными результатами (см., например $\left.\left[{ }^{13}\right]\right)$. В шкале энергии перехода вращательного движения $\Delta E^{\text {вращ }}$ распределение вероятностей $\left.\left|M_{f}^{\text {враш }}\right|\right|^{2}$ имеет вид

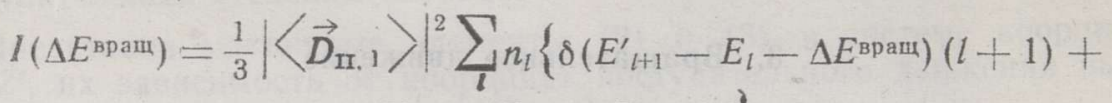

$$
\begin{aligned}
& \left.+\delta\left(E_{l-1}^{\prime}-E_{l}-\Delta E^{\text {враш }}\right) l\right\} \text {, }
\end{aligned}
$$

где $E_{l}=\hbar^{2} l(l+1) / 2 I_{1} ; \quad E_{l}^{\prime}=\hbar^{2} l(l+1) / 2 I_{\mathrm{II}} ; \quad$ для линейных молекул $n_{l}=N \exp \left(-E_{l} / k T\right)$, для молекул типа сферического волчка $n_{l}=N(2 l+1) \exp \left(-E_{l} / k T\right)$.

Первая сумма в (16) определяет вероятности вращательных -переходов в $R$-ветви (с изменением орбитального квантового числа $l$ на величину $\Delta l=1)$, вторая - в $P$-ветви $(\Delta l=-1)$. Функции распределения вероятностей перехода в каждой ветви обычно имеют огибающую с одним максимумом. Энергия перехода с максимальной вероятностью $E_{w}$ соответствует приблизительно исходному энергетическому уровню наиболышей заселенности. В случае молекул типа сферического волчка эти энергии перехода следующие:

$$
\left.\begin{array}{c}
E_{\omega}(R)=\hbar \sqrt{\frac{2 k T}{I_{\mathrm{I}}}}+\left(\frac{I_{\mathrm{I}}}{I_{\mathrm{II}}}-1\right) k T \\
E_{\omega}(P)=-\hbar \sqrt{\frac{2 k T}{I_{\mathrm{I}}}}+\left(\frac{I_{\mathrm{I}}}{I_{\mathrm{II}}}-1\right) k T
\end{array}\right\} .
$$

Таким образом, расстояние между максимумами $R$ - и $P$-ветвей равно приблизительно $2 \hbar \sqrt{2 k T / I_{\mathrm{I}}}$. Оценка величины этого расстояния приведена на рисунке.

Для внутриядерного перехода будем вычислять матричные элементы $M_{f i}^{\text {враш }}$ в случае поглощения (излучения) достаточно малых частот линейными молекулами или молекулами типа сферического волчка.

Ограничиваясь первыми двумя членами в разложении (3), получим!

$$
M\left(X^{\text {вращ })} \sim 1+i \frac{\omega}{c}\left\langle R_{L}^{\prime}\right\rangle \cos \vartheta,\right.
$$


где $\left\langle R_{L}^{\prime}\right\rangle$ означает матричный элемент по колебательным состояниям длины радиус-вектора ядра номера $L$. Распределение вероятностей $\left|M_{f i}^{\text {вращ }}\right|^{2}$ (сравни также с $\left.\left.{ }^{[14}\right]\right)$ для молекул типа сферического волчка будет следующее:

$$
\begin{aligned}
& I\left(\Delta E^{\text {вращ }}\right)=\sum_{l} n_{l}\left\{\delta\left(E_{l}^{\prime}-E_{l}-\Delta E^{\text {вращ }}\right)(2 l+1)+\right. \\
& +\frac{\omega^{2}}{3 c^{2}}\left\langle R_{L}^{\prime}\right\rangle^{2} \delta\left(E_{l+1}^{\prime}-E_{l}-\Delta E^{\text {вращ }}\right)(l+1)+ \\
& \left.+\frac{\omega^{2}}{3 c^{2}}\left\langle R_{L}^{\prime}\right\rangle^{2} \delta\left(E_{l-1}^{\prime}-E_{l}-\Delta E^{\text {вращ }}\right) l\right\} .
\end{aligned}
$$

Здесь первое слагаемое в фигурных скобках определяет вероятности вращательных переходов в $Q$-ветви $(\Delta l=0)$, второе - в $R$-ветви $(\Delta l=1)$ и третье - в $P$-ветви $(\Delta l=-1)$. Энергия перехода с максимальной вероятностью в $Q$-ветви прнблизительно $E_{w}(Q)=\left[\left(I_{\mathrm{I}} / I_{\mathrm{II}}\right)-\right.$ - 1]kT, для $R$ - и $P$-ветвей эти энергии уже оценены в (17). Таким образом, изменение момента инерции молекулы в переходе смещает вращательный спектр на величину порядка $\left[\left(I_{\mathrm{I}} / I_{\mathrm{II}}\right)-1\right] k T$ и тем самым приводит к уширению $Q$-ветви на величину такого же порядка. Например, изменение момента инерции молекулы на $1 \%$ приводит при комнатной температуре к уширению $Q$-ветви на величину порядка $2 \mathrm{~cm}^{-1}$. Из (19) видно, что отношение суммарных вероятностей $R$ - и $P$-ветвей к суммарной вероятности $Q$-ветви равно $\omega^{2}\left\langle R_{L}^{\prime}\right\rangle^{2} / 3 c^{2}$.

\section{4. Колебательное движение}

Влиянием поступательного и вращательного движений пока пренебрежем и рассмотрим сначала переход в электронной оболочке молекулы.

Небольшая молекула имеет набор нормальных частот, среди которых нет ни очень малых частот, ни большого числа частот на узком частотном интервале. Колебательная структура спектра состоит из более или менее хорошо разрешенных отдельных линий, ширина которых определяется шириной (временем жизни) соответствующих переходу колебательных подуровней. Колебательная структура наиболее четка около чистоэлектронной линии, причем сама чистоэлектронная линия является наиболее узкой и обладает в отсутствие безызлучательных переходов в другие электронные состояния радиационной шириной. Благодаря чрезвычайной узости $\left(10^{-4} \mathrm{~cm}^{-1}\right.$ для разрешенных переходов, егце меньше для запрешенных) интенсивность линии в максимуме весьма велика. Интегральная интенсивность чистоэлектронной линии тем меньше, чем больше стоксовы потери и чем выше температура. Однако, главная цель нашего рассмотрения - большие молекулы. Тогда среди нормальных колебаний имеются коллективные движения, напоминающис длинноволновые колебания в кристалле, и обладающие настолько густым спектром частот $\omega_{ф}$, что разности $\delta \omega_{ф}$ между ними порядка уширения $\Delta \omega_{\phi}$ каждой из них. В этом случае возникает вопрос об уширении чистоэлектронной линии электронно-колебательными переходами, в которых рождаются или уничтожаются колебательные кванты весьма малых частот. Возникает задача, весьма близкая к задаче об уширении акустическими колебаниями чистоэлектронной линии в спектре примесного центра в кристалле. Если электронное воз буждение локализовано на сравнительно небольшой группе атомов, то 
почти непосредственно применима теория квазилинейчатых электронноколебательных спектров (см., например $[15,2,3]){ }^{*}$. Остается в силе основной результат этой теории: из-за специфики распределения стоксовых потерь между колебаниями молекулы переходы с изменением числа квантов $\hbar \omega_{ф}$ колебаний не уширяют чистоэлектронную линию, а при. ведут к появлению колебательного фона около нее. Чистоэлектронная линия выделяется в виде весьма узкого пика на фоне спектра, обсуслов. ленного переходами с изменением числа колебательных квантов. Ее интегральная интенсивность убывает быстро (экспоненщиально) с ростом стоксовых потерь и температуры.

Все сказанное относится также к линии Мессбауэра, оптическим аналогом которой является чистоэлектронная линия.

Если обе линии рассматриваются в приближении описания колебаний гармоническими осцилляторами, у которых в результате перехода изменяются только положения равновесия, то формулы, описывающие поведение линии Мессбауэра, получаются из соответствующих формул теории чистоэлектронной линии, если в последних стоксовы потери заменить на энергию отдачи $\gamma$-кванта $\left[{ }^{1,}, 3\right]$. Аналогия между явлениями сохраняется и в более точных вариантах теории: задача о распределении энергии отдачи $\gamma$-кванта между колебаниями может рассматриваться в импульсном пространстве как задача о распределении стоксовых потерь между колебаниями [8].

Вспомним теперь о поступательном (допплеровском) уширения линии, которое, как мы увидели, при достаточно тяжелых молекулах будет преобладать над вращательным уширением. Оно намного превышает радиационную ширину. Это обстоятельство весьма существенно для толкования чистоэлектронной линии (линии Мессбауэра) и колебательной структуры спектра в ее окрестности: в наблюдаемую интенсивность этой линии надо теперь включить и интенсивность фона на участке протяженности порядка допплеровского уширения, т. е. на интервале частот, превышающем на несколько по рядков радиационную ширину. Вклад фона особенно существеңен при высоких температурах, когда становятся возможными переходы, в которых одновременно рождаются и уничтожаются колебательные кванты близких частот. Так как большие молекульт могут существовать в газовой фазе только при достаточно высоких температурах, то в нашей задаче это необходимо учесть. В результате наблюдаемая чистоэлектронная линия будет иметь иную температурную зависимость интенсивности, чем собственно чистоэлектронная линия, причем эффективная ширина ее определяется вовсе не колебаниями, а допплеровским уширением.*

Для нашей основной задачи - выяснения возможностей проявления чнстоэлектронной линии (линии Мессбауэра) в спектрах больших молекул, важно, что колебания не прнводят к дополнительному уширению этих линий.

- Вспомним, что мы с самого начала рассматриваем переход между парой электронных уровней, энергетнчески хорошо изолированных от остальных электронных уровней. Если возбужденному состоянию соответствует электронное возбуждение, распространяющееся на всю большую молекулу, то следует думать, что следующие электронные уровни лежат близко (уровни свободного электрона в потенциальном ящнке большого размера согласно металлической модели молекулы). Поэтому. нанболее соответствующие модели объекты - это именно такие молекулы, где наинизший возбуж. денный электронный уровень соответствует возбуждению сравнительно небольшой части молекулы. Қроме того, если же некоторый энергетически нзолнрованный уровень соответствует электронному возбуждению, распространяющемуся на всю молекулу, то электронный переход вообще весьма мало влияет на ее колебания (малые суммарные стоксовы потери) и проблема колебательного ушнрения становится не очень актуальной.

** В крнсталлах, которые могут рассматриваться как молекулы с макроскопнческой массой, допплеровскюе уширение становится ннчтожно малым, линия Мессбауэра достигнет раднационной ширины, а чистоэлектронная линия остается уширенной до $0,1-1$ с $^{-1}$ из-за неоднородностей кристаллнческой матрицы [16]. Можно однако нацеяться, что методика квантовых биений $\left[{ }^{17}\right]$ позволяет освободиться от этого уширения и экспернментально обнаружить нстинную ширину чистоэлектронных линий. 


\section{5. Обсуждение результатов}

Сравнение уширения спектральной линии за счет поступательного и врашательного движений молекулы (если принимать за меру последнего уширения расстояние между максимумами $R$ - и $P$-ветвей) показывает, что оба уширения пропорциональны $\sqrt{k T / m}$, при этом ушкрение за счет поступательного движения еще пропорционально энергии поглощающегося (излучающегося) кванта, а уширение за счет вращательного движения обратно пропорционально радиусу инерции молекулы. Поэтому последнее уширение уменьшается быстрее с увеличением массы и при достаточно тяжелых молекулах будет преобладать первое уширение (см. рисунок). Отношение первого уширения ко второму равно $\sqrt{\ln 2} r_{\mathrm{I}} \times$ $X \omega / c\left(r_{\mathrm{T}}-\right.$ радиус инерции молекулы). Уширение $Q$-ветви пропорционально $k T$ и нзменению момента инерции молекулы.

$\mathrm{Ha}$ рисунке приведено

Зависимость обусдовленной посту пательным движением полуширины спектральной линии $\delta$ от массы молекулы для различных величиі поглощающихся (излучающихся) квантов (кривые 1), обусловленное энергией отдачи смещение $2 E_{R}$ ли нии поглощения относительно линии излучения в зависимости от массы молекулы для различных величин квантов (кривые 2) и расстояние между максимумами $R$ Р-ветвей $E_{w}(R)-E_{w}(P)$ в завнсимости от массы молекулы

(крнвая 3). Кривые даны при комнатной температуре.

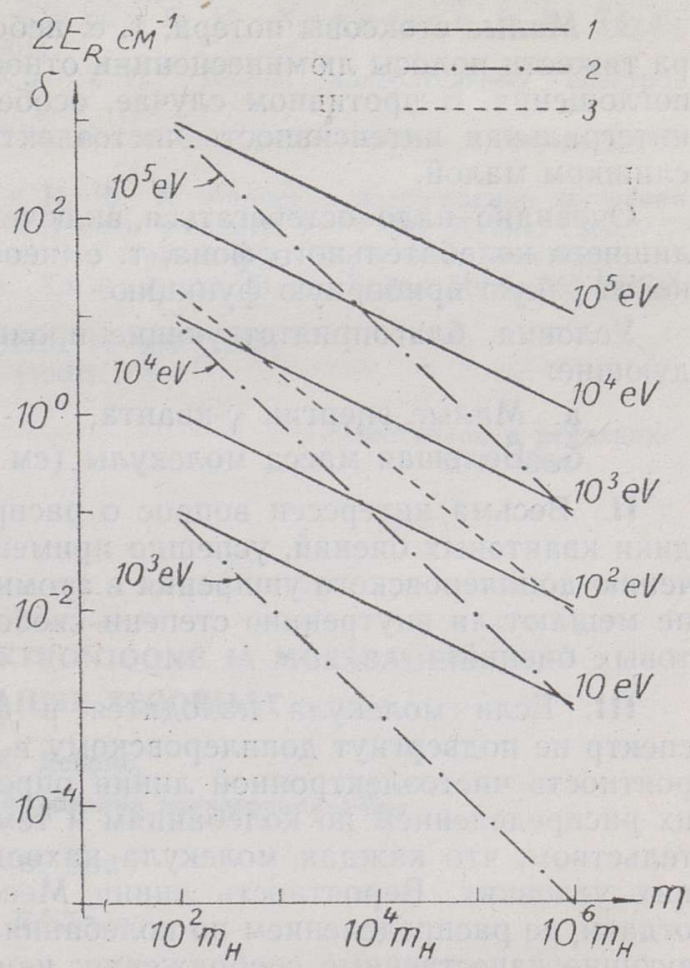

уширение спектральной линии при комнатной температуре за счет поступательного движения молекулы в зависимости от массы молекулы $m$ при разных энергиях поглощающихся (излучающихся) квантов (см. рисунок, кривые 1). Кривая 3 дает уширение спектральной линии за счет вращательного движения молекулы, при условии, что молекула считается шариком с однородно распределенной массой плотности $5 \mathrm{c} / \mathrm{CM}^{3}$.

Нз предыдущего можно заключить, что наблюдение электронно-колебательных спектральных линий, ширины меньше $1 \mathrm{~cm}^{-1}$, возможно при комнатной температуре у молекул с массой больше $10^{4} m_{H}$. Для наблюдения ядерно-колебательных спектральных линий такой же ширнны пригодны в случае сверхмягких $ү$-квантов такие же молекулы, а в случае более жесткнх $\gamma$-квантов нужно подобрать соответственно (см. рисунок) более тяжелые молекулы. Наблюдение более узких (шириной в $10^{-3} \mathrm{CM}^{-1}$ ) ядерно-колебательных спектральных линий (линии Мессбауэра) возможно уже в случае сверхмягких $\gamma$-квантов только у невероятно больших молекул с массой больше $10^{10} \cdot m_{H}$. 


\section{6. Заключительные замечания}

1. Сформулируем еще раз условкя, благоприятствующие проявлению чистоэлектронной линии в спектрах поглощения и люминесценцин свободных молекул:

1. Достаточная энергетическая изоляция электронных уровней Вполне достаточным может считаться, по-видимому, расстояние порядка $1000 \mathrm{CM}^{-1}$

2. Достаточно большая масса. При $m=10^{3} m_{H}$ уширение от вращения и поступательного движения молекулы менее $3 \mathrm{~cm}-1$, т. е. уширение такого же порядка, как в примесных спектрах кристаллов.

3. Малые стоксовы потери, т. е. небольшое стоксово смещение центра тяжести полосы люминесценции относительно центра тяжести полосы поглощения. В противном случае, особенно при высоких температурах, интегральная интенсивность чистоэлектронной линии может оказаться слишком малой.

Очевидно надо остерегаться включения в чистоэлектронную линию лишнего колебательного фона, т. е. необходимо обеспечить по возможности узкую приборную функцию.

Условия, благоприятствующие проявлению линии Мессбауэра, следующие:
а. Малые энергии $\gamma$-кванта.
б. Большая масса молекулы (см. рисунок).

II. Весьма интересен вопрос о распространении на молекулы методики квантовых биений, успешно примененной Александровым к исключению допплеровского уширения в атомных спектрах [17]. Однако неясно, не мешают ли внутренние степени свободы молекулы реализации квантовых биений.

III. Если молекула находится в адсорбированном состоянии, ее спектр не подвергнут допплеровскому и вращательному уширениям. Вероятность чистоэлектронной линии определяется стоксовыми потерями, их распределением по колебаниям и температурой, ширина тем обстоятельством, что каждая молекула находится в слегка различных внешних условиях. Вероятность линии Мессбауэра определяется энергией отдачи, ее распределением по колебаниям и температурой. Полезны следующие качественные соображения: чем меньше размеры объема (выраженные в подходящих безразмерных единицах, например, в амплитудах нулевого колебания), в котором сконцентрирована квантовомеханическая вероятность пребывания взаимодействующего с $\gamma$-квантом ядра в ходе колебаний перед $\gamma$-переходом, тем более интенсивна при данной энергии отдачи линия Мессбауэра*. Важно, что совсем необязательна «жесткая» связь $y$-активного ядра с кристаллом.

IV. Любопытным объектом для изучения влияния вращения на линию Мессбауэра являются кристаллы со степенями свободы внутреннего вращения.

Авторы искренне благодарны В. В. Хижнякову за обсуждение работы.

* Это правило вытекает из рассмотрения Фурье-образа функции распредеяе ния коордннат колебания $\gamma$-активного ядра (см., например $\left.\left[{ }^{18}\right]\right)$. 
1. Т р и фоно в Е. Д., ДАН СССР, 147, № 4, 826 (1962).

2. Реб ане К. К., Хи жняков В. В., Опт. и спектр., 14, 362 (1963).

3. Ребане К. К., Хи жняков В. В., Опт. и спектр., 14, 491 (1963),

4. Гросс Е. Ф., Разбирин. Б. С., Перм огоров С. А., ДАН СССР, 147, 338 (1962).

5. Ш польский Э. В., УФН, 71, $215(1960) ; 77,321$ (1962).

6. Ш п ольский Э. В., УФН, 80, 254 (1963).

7. С теп анов Б. И., Люминесценция сложных молекул, Минск, 1956.

8. Р еб ане Қ. К., Опт. и спектр., 16, 594 (1964).

9. L a x M., J. Chem. Phys., 20, 1752 (1952), (см. перевод в сб. Проблемы физики полупроводников, ИЛ, М., 1957).

10. Р е б а н е К. К., Опт. и спектр., 9, 557 (1960).

11. Ребане К. К., Пурга А. П., Сильд О. И., Хижняков В. В., Труды ИФА АН ЭССР, № 14, 31 (1961).

12. Вильсон Е., Деши ус Дж., Кросс П., Теорня колебательных спектров молекул, Ил, М., 1960.

13. Бете Г., С олпитер Э., Квантовая механика атомов с одним и двумя электронами, ГИФМЛ, М., 1960.

14. Подгорещки й $\mathrm{M}$. И., $\mathrm{P}$ ойз ен И. И., $\mathrm{K}$ вопросу о возбуждении вращения молекулы за счет отдачи при излучении $ү$-кванта, Р - 592, Дубна, 1960.

15. Кривоглаз М. А. и Пекар С. И., Труды ИФ уССР, в. 4,37 (1953).

16. Ребане К. Қ., Техвер И. Ю., Хижняков В. В., Труды ИФА АН ЭССР. № 27,17 (1964).

17. Алекс андров Е. Б., Опт. и спектр., 14, 436 (1963)

18. Lipkin H. J., Ann. Phys., 18, 182 (1962).

Институг физики и астрономии

Академии наук Эстонской ССР
Поступила в редакцию

5. II 1964

\title{
VABA MOLEKULI ELEKTRONJOONE JA MOSSBAUERI JOONE LAIUSE TEOORIAST
}

\author{
K. Rebane, \\ Eesti NSV Teaduste Akadeemia korrespondentliige
}

o. Sild

\section{Resümee}

Artiklis uuritakse kulgliikumise, pöörlemise ja võnkumise mõju elektronjoone ja Mössbaueri joone laiusele vaba molekuli spektris. Näidatakse, et kulgliikumisest ja pöörlemisest tingitud spektraaljoone laius väheneb molekuli massi $m$ suurenemisel. Kulgliikumisest tingitud spektraaljoone laius on võrdeline suurusega $\sqrt{k T / m}(T-$ absoluutne temperatuur) ja neelduva või kiirguva kvandi energiaga. Pöörlemisest tingitud spektraaljoone laius on võrdeline suurusega $\sqrt{k T / m}$ ja pöördvõrdeline molekuli inertsiraadiusega. Küllaldaselt suure massiga molekulide spektraaljoone laius on seetõttu määratud kulgliikumisega.

Vaba molekuli spektri võnkestruktuur sarnaneb kristalli luminestsentsitsenntri spektri võnkestruktuuriga. Sellest järeldub, et elektronjoone või Mössbaueri joone suhteline intensiivsus spektris väheneb kiiresti (eksponentsiaalselt) Stokes'i kadude või põrkeenergia suurenemisel.

Elektronjoont (laiusega $<10 \mathrm{~cm}^{-1}$ ) on võimalik vaadelda järgmistel tingimustel: 1) entrgeetiliselt isoleeritud elektronnivood; 2) väikesed Stokes'i kaod ja 3) küllaldaselt suure massiga molekulid $\left(>10^{3} m_{H}\right.$, kui $m_{H}$ on vesinikuaaatomi mass). Mössbaueri joone (laiusega $<10^{-3} \mathrm{~cm}^{-1}$ ) vaatlemiseks on vaja valida isegi väga pehmete $\gamma$-kvantide korral äärmiselt suure massiga $\left(>10^{10} m_{H}\right)$ molekulid. 


\title{
ZUR THEORIE DER ELEKTRONENLINIE UND DER MÖSSBAUER-LINIE FREIER MOLEKULE
}

\author{
K. Rebane, \\ Korrespondierendes Mitglied der Akademie der Wissenschaften \\ der Estnischen SSR
}

0. Sild

Zusammenfassung

In vorliegender Arbeit wird der Einfluss der translatoren, der rotatoren und der Schwingungsbewegung auf die Breite der reinen Elektronenlinie und der MössbauerLinie im Spektrum freier Moleküle untersucht. Es wird gezeigt, dass die von der translatoren und der rotatoren Bewegung bedingte Linienbreite mit dem Wachsen der Molekülmasse $m$ abnimmt. Die von der translatoren Bewegung herrührende Linienbreite ist proportional zu $\sqrt{k T / m}(T$ - die absolute Temperatur) und der Energie absorbierter oder emittierter Quanten. Die von der Rotation bedingte Linienbreite ist gleichfalls proportional zu $\sqrt{k T / m}$ und umgekehrt proportional zum Trägheitsradius des Moleküls, Die Linienbreite im Spektrum genügend massiver Moleküle wird demnach durch die translatore Bewegung bestimmt.

Die Schwingungsstruktur im Spektrum freier Moleküle ist derjenigen des Lumineszenzzentrums im Kristall ähnlich. Daraus lässt sich schliessen, dass die relative Intensität der reinen Elektronen- oder Mössbauer-Linie mit dem Wachsen der StokesVeriuste (oder mit dem Wachsen der Rückstossenergie) schnell (exponentiell) abnimmt.

Die reine Elektronenlinie (Halbbreite $<10 \mathrm{~cm}^{-1}$ ) lässt sich unter folgenden Bedingugen beobachten: 1) energetisch genügend isolierte Elektronenniveaus, 2) kleine Stokes-Verluste, 3) genügend massive Moleküle $\left(>10^{3} m_{H}\right.$, wenn $m_{H}$ die Masse des Wasserstoffatoms bedeutet). Zur Beobachtung der Mössbauer-Linie (Halbbreite $\left\langle 10^{-3} \mathrm{~cm}^{-1}\right)$ sind sogar für sehr weiche $\gamma$-Quanten äusserst massive Moleküle $\left(>10^{10} m_{H}\right)$ notwendig.

Institut für Physik und Astronomie der Akademie der Wissenschaften der Estnischen SSR
Eingegangen am 5. Febr. 1964 